\title{
Viral genomes are part of the phylogenetic tree of life
}

\section{Ethan B. Ludmir and Lynn W. Enquist}

In their recent review Moreira and LópezGarcía presented ten reasons to exclude viruses from the tree of life, with the fundamental assertion that 'viruses are not alive' (Ten reasons to exclude viruses from the tree of life. Nature Rev. Microbiol. 7, 306-311 (2009)) $)^{1}$. This assertion is an oversimplification. Virions (physical virus particles) indeed are dead: they are inert and are driven solely by thermodynamically spontaneous reactions ${ }^{2}$. Yet, the phylogenetic tree is based on the genomic content of its component organisms, not the physical manifestations of these genomes ${ }^{3}$. Nucleotide sequences alone place a genome in the phylogenetic tree. Accordingly, the question becomes: should viral genomes be included in this tree? We think so.

Viral genomes are differentiated from other replicons and genetic material, such as viroids or plasmids, through a unique threepart strategy for survival that is common to all viruses. First, viral genomes are packaged into proteinaceous particles (virions). Second, the viral genome encodes all the necessary information to allow completion of an infectious cycle with a single cell, from attachment and entry into a host cell to replication of progeny to egress. Third, the viral genome encodes gene products that ensure its stable propagation and maintenance in a host population. This tripartite strategy separates viral genomes from other genetic material that does not encode a cellular organism.

Moreira and López-García maintain that cellular organisms are capable of selfmaintenance and self-replication, but that viruses are not ${ }^{1}$. Yet they include cellular obligate intracellular parasites, such as Chlamydia and Rickettsia, in the tree of life ${ }^{4}$. This distinction was made because, unlike any viral genome, cellular intracellular parasites encode their own ribosomes. It is not clear why this fact precludes viral genomes from holding a place in the tree of life. If anything, the absence of remnants of ribosome-encoding genetic material in viruses highlights the evolutionary symbiosis of viral and cellular genomes on a grander scale. It is difficult to imagine life without viruses; indeed, viral genomes have been implicated in many major evolutionary milestones, from the introduction of DNA into the RNA world to the appearance of a nucleus ${ }^{5-7}$.

Moreira and López-García also assert that the polyphyletic nature of viruses and the absence of any known ancestral viral lineages is sufficient reason to exclude their genomes from the tree of life ${ }^{1}$. This assertion discounts one of the most powerful attributes of viral genomes: mutation rates that are orders of magnitude higher than those for most cellular genomes ${ }^{2}$. This attribute increases the probability that new genomes will survive virtually any selection and enables a viral population to survive over a large span of time. Although it is difficult to determine common ancestors for viral families that diverged countless generations ago, both sequence homology and structural homology suggest that RNA and DNA viral genomes stem from two independent lineages ${ }^{8}$. Accordingly, viral genomes do meet the Moreira and LópezGarcía criteria: homology between members of each of these two lineages forms the basis for common ancestry ${ }^{1}$.

However, Moreira and López-García dismiss these arguments on the grounds that convergent evolution accounts for common capsid symmetry and that horizontal gene transfer (HGT) complicates reconstruction of viral phylogenies ${ }^{1}$. Although convergence indeed might account for the maintenance of capsid symmetry rules across a broad range of virions, non-structural gene products, particularly replication proteins, are far more important to the determination of phylogenies. Homologies among these central units of genome replication provide evidence for common viral ancestry ${ }^{8}$. Additionally, HGT is not likely to be important in viral genome evolution as only a small fraction of viral proteins have been suggested as possible viral acquisitions from cells through gene transfer ${ }^{8}$. The sequencing of the Mimivirus genome revealed a small number of cellularderived metabolic genes ${ }^{9}$. Furthermore, approximately two-thirds of predicted protein-coding genes ( 600 open reading frames (ORFs)) in the Mimivirus genome have no functional similarity to any known proteins, suggesting that Mimivirus diverged early in evolution'. This finding is by no means exclusive to Mimivirus; approximately $60 \%$ of the ORFs of the bacteriophage sk1 genome have no known functional homologues $^{10}$, as is the case with $94 \%$ of the white spot syndrome virus genome ORFs ${ }^{11}$.

We propose that four criteria should be considered when determining whether evolving genetic material is part of the phylogenetic tree. Genomes and their gene products must be able to produce progeny genomes, possess internal regulation, adapt and respond to changing environmental conditions, and maintain structural organization; in other words, the genomes must be capable of reproduction, self-regulation, adaptation and structural maintenance. The first criterion, the ability to reproduce, is a property shared by all replicons; therefore, the other three criteria, self-regulation, adaptation and maintenance, differentiate viral and cellular genomes from other replicating genetic material.

Expression of information in viral genomes is highly regulated. Most DNA viruses, for example, have a timing mechanism for differential gene transcription that is based on the replication cycle (for example, early or late) ${ }^{2}$. Similarly, Mononegavirales genomes use regulation of the viral RNA-dependent RNA polymerase to differentiate synthesis of mRNA and replication of the genome ${ }^{12}$. All viral genomes have evolved complex techniques for internal regulation (homeostasis), such as genome polarity, transcriptional activators and protease modification of viral polymerases.

Adaptation and responsiveness, which are inherently difficult qualities to assign to genomes with short replicative cycles, are nevertheless attributes of information encoded in viral genomes. Although virions themselves are 'dead', the viral genomes carried inside virions encode gene products that allow for adaptation and response to changing intracellular and extracellular conditions. In particular, these gene products enable adaptation to host antiviral defences. Two striking examples of responsiveness are Herpesviridae reactivation from latency, a process that relies on cellular cues ${ }^{13}$, and prophage reactivation in lysogenic bacteria after they receive signals of impending host cell death ${ }^{14}$.

Finally, Moreira and López-García assert that viruses are examples of structural simplicity ${ }^{1}$. One need not look long at the gallery of virions to see structural diversity, 
not simplicity. Even in those virions that share basic icosahedral and helical capsid symmetry, remarkable complexity has evolved to promote viral propagation. A prime example of this is Orthoreovirus, for which structural complexity of the virion (double layer capsid, replication and mRNA synthesis machinery at each of the 12 vertices) facilitates propagation of the double-stranded RNA (dsRNA) genome without activating intrinsic host cell defences against cytosolic dsRNA ${ }^{15}$. Consider the Fullerene cone capsids of HIV-1, the multi-membrane development of the vaccinia virion and the amazing architecture of archaeal virions that survive in ultra-extreme conditions, as well as the well-known bacteriophage $\mathrm{T} 4$ virion; all are testament to the reality of structurally complex, diverse and functional systems to protect and deliver genomes.

Viral genomes represent essential elements of the phylogeny of life and have played important parts in evolution. Viral genomes meet the proposed four criteria for genomic classification within the phylogenetic tree, are not polyphyletic and are capable of being traced back through two lineages. Above all, virions carry unique genetic material, unified by a common tripartite replication strategy. The intricacies of each strategy, yet the common outcome, are testament to the distinctive character of viruses and justify their inclusion into the phylogenetic tree.

Ethan B. Ludmir and Lynn W. Enquist are at the Department of Molecular Biology, Princeton University, Princeton, 08544 New Jersey, USA. e-mail: lenquist@Princeton.EDU

1. Moreira, D. \& López-García, P. Ten reasons to exclude viruses from the tree of life. Nature Rev. Microbiol. 7, 306-311 (2009).

2. Flint, S. J., Enquist, L. W., Racaniello, V. R. ¿ Skalka, A. M. in Principles of Virology, Third Edition (ASM Press, Washington DC, 2009).

3. Doolittle, W. F. Phylogenetic classification and the universal tree. Science 284, 2124-2128 (1999)

4. Zomorodipour, A. \& Andersson, S. G. E. Obligate intracellular parasites: Rickettsia prowazekii and Chlamydia trachomatis. FEBS Lett. 452, 11-15 (1999).

5. Bell, P. J. Viral eukaryogenesis: was the ancestor of the nucleus a complex DNA virus? J. Mol. Evol. 53, 251 (2001).

6. Forterre, P. The two ages of the RNA world, and the transition to the DNA world: a story of viruses and cells. Biochimie 87, 793-803 (2005).
7. Takahashi, I. \& Marmur, J. Replacement of thymidylic acid by deoxyuridylic acid in the deoxyribonucleic acid of a transducing phage for Bacillus subtilis. Nature 197, 794-795 (1963).

8. Forterre, P. The origin of viruses and their possible roles in major evolutionary transitions. Virus Res. 117 5-16 (2006).

9. Raoult, D. et al. The 1.2-megabase genome sequence of Mimivirus. Science 306, 1344-1350 (2004).

10. Chandry, P. S., Moore, S. C., Boyce, J. D., Davidson, B. E. \& Hillier, A. J. Analysis of the DNA sequence, gene expression, origin of replication and modular structure of the Lactococcus lactis lytic bacteriophage sk1. Mol. Microbiol. 26, 49-64 (1997).

11. Van Hulten, M. C. W. et al. The white spot syndrome virus DNA genome sequence. Virology 286, 7-22 (2001).

12. Whelan, S. P. J., Barr, J. N. \& Wertz, G. W. Transcription and replication of nonsegmented negative-strand RNA viruses. Curr. Top. Microbiol. Immunol. 283, 61-119 (2004).

13. Liu, T., Khanna, K. M., Chen, X., Fink, D. J. \& Hendricks, R. L. CD 8 ${ }^{+} \mathrm{T}$ cells can block herpes simplex virus type 1 (HSV-1) reactivation from latency in sensory neurons. J. Exp. Med. 191, 1459-1466 (2000).

14. Panet, A., Braun, E., Honigman, A. \& Steiner, I. Involvement of cellular death signals in the reactivation of herpes simplex virus type 1 and lambda bacteriophage from a latent state. J. Theor. Biol. 236, 88-94 (2005).

15. Reinisch, K. M., Nibert, M. L. \& Harrison, S. C. Structure of the reovirus core at $3.6 \AA$ resolution. Nature 404, 960-967 (2000).

Acknowledgements

The authors thank M.P. Taylor, M.G. Lyman, O. Kobiler, T. Kramer and H. Prag Naveh for insightful comments regarding this correspondence. 